\title{
Correction to: Viscosity Measurements of Dialkyl Adipates in the Temperature Range of (283 to 363) K and up to $40 \mathrm{MPa}$
}

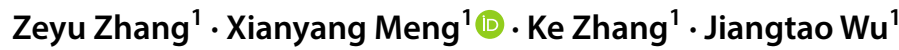

Published online: 27 March 2020

(C) Springer Science+Business Media, LLC, part of Springer Nature 2020

\section{Correction to: International Journal of Thermophysics (2019) 40:109 https://doi.org/10.1007/s10765-019-2573-6}

The original version of the article unfortunately contained some errors in Table 3. The viscosity data of diethyl adipate from $353.15 \mathrm{~K}$ and $40 \mathrm{MPa}$, to $363.15 \mathrm{~K}$ and $40 \mathrm{MPa}$ were modified. Corrected version of Table 3 is given below.

Selected Papers of the 14th International Symposium on Temperature and Thermal Measurements in Industry and Science

The original article can be found online at https://doi.org/10.1007/s10765-019-2573-6.

Xianyang Meng

xymeng@mail.xjtu.edu.cn

1 Key Laboratory of Thermo-Fluid Science and Engineering, Ministry of Education, School of Energy and Power Engineering, Xi' an Jiaotong University, Xi' an 710049, China 
Table 3 Viscosity $\eta$ for Diethyl Adipate at Different

Temperatures $T$ and Pressures $p$ up to $40 \mathrm{MPa}$ Measured with the Vibrating Wire Technique

\begin{tabular}{|c|c|c|c|}
\hline$T / \mathrm{K}$ & $p \cdot \mathrm{MPa}^{-1}$ & $\rho_{\mathrm{cal}}^{\mathrm{a}} \cdot \mathrm{kg}^{-1} \cdot \mathrm{m}^{3}$ & $\eta_{\exp } \cdot \mathrm{mPa}^{-1} \cdot \mathrm{s}^{-1}$ \\
\hline 283.15 & 0.1 & 1015.85 & 4.5405 \\
\hline 283.15 & 1 & 1016.42 & 4.5895 \\
\hline 283.15 & 5 & 1018.94 & 4.7921 \\
\hline 283.15 & 10 & 1022.02 & 5.0621 \\
\hline 283.15 & 15 & 1025.01 & 5.3319 \\
\hline 283.15 & 20 & 1027.92 & 5.6216 \\
\hline 283.16 & 30 & 1033.52 & 6.2229 \\
\hline 283.15 & 40 & 1038.88 & 6.8866 \\
\hline 293.15 & 0.1 & 1006.51 & 3.4862 \\
\hline 293.15 & 1 & 1007.12 & 3.5210 \\
\hline 293.15 & 5 & 1009.78 & 3.6723 \\
\hline 293.15 & 10 & 1013.01 & 3.8679 \\
\hline 293.15 & 15 & 1016.15 & 4.0747 \\
\hline 293.15 & 20 & 1019.21 & 4.2783 \\
\hline 293.15 & 30 & 1025.08 & 4.7218 \\
\hline 293.15 & 40 & 1030.66 & 5.1950 \\
\hline 303.15 & 0.1 & 997.15 & 2.7663 \\
\hline 303.15 & 1 & 997.79 & 2.7923 \\
\hline 303.15 & 5 & 1000.60 & 2.9116 \\
\hline 303.15 & 10 & 1004.00 & 3.0629 \\
\hline 303.15 & 15 & 1007.30 & 3.2068 \\
\hline 303.15 & 20 & 1010.51 & 3.3610 \\
\hline 303.15 & 30 & 1016.65 & 3.6955 \\
\hline 303.15 & 40 & 1022.48 & 4.0422 \\
\hline 313.15 & 0.1 & 987.76 & 2.2525 \\
\hline 313.16 & 1 & 988.43 & 2.2717 \\
\hline 313.15 & 5 & 991.40 & 2.3617 \\
\hline 313.15 & 10 & 994.98 & 2.4781 \\
\hline 313.15 & 15 & 998.45 & 2.5970 \\
\hline 313.15 & 20 & 1001.82 & 2.7206 \\
\hline 313.15 & 30 & 1008.25 & 2.9780 \\
\hline 313.15 & 40 & 1014.33 & 3.2507 \\
\hline 323.15 & 0.1 & 978.35 & 1.8698 \\
\hline 323.15 & 1 & 979.06 & 1.8858 \\
\hline 323.15 & 5 & 982.18 & 1.9606 \\
\hline 323.15 & 10 & 985.96 & 2.0537 \\
\hline 323.15 & 15 & 989.61 & 2.1509 \\
\hline 323.15 & 20 & 993.14 & 2.2485 \\
\hline 323.15 & 30 & 999.87 & 2.4607 \\
\hline 323.15 & 40 & 1006.22 & 2.6798 \\
\hline 333.15 & 0.1 & 968.90 & 1.5793 \\
\hline 333.15 & 1 & 969.66 & 1.5923 \\
\hline 333.15 & 5 & 972.96 & 1.6529 \\
\hline
\end{tabular}


Table 3 (continued)

\begin{tabular}{|c|c|c|c|}
\hline$T / \mathrm{K}$ & $p \cdot \mathrm{MPa}^{-1}$ & $\rho_{\mathrm{cal}}^{\mathrm{a}} \cdot \mathrm{kg}^{-1} \cdot \mathrm{m}^{3}$ & $\eta_{\exp } \cdot \mathrm{mPa}^{-1} \cdot \mathrm{s}^{-1}$ \\
\hline 333.15 & 10 & 976.93 & 1.7335 \\
\hline 333.15 & 15 & 980.77 & 1.8143 \\
\hline 333.15 & 20 & 984.47 & 1.8958 \\
\hline 333.15 & 30 & 991.52 & 2.0658 \\
\hline 333.15 & 40 & 998.15 & 2.2487 \\
\hline 343.15 & 0.1 & 959.43 & 1.3538 \\
\hline 343.15 & 1 & 960.23 & 1.3672 \\
\hline 343.15 & 5 & 963.71 & 1.4189 \\
\hline 343.15 & 10 & 967.90 & 1.4873 \\
\hline 343.15 & 15 & 971.94 & 1.5546 \\
\hline 343.15 & 20 & 975.82 & 1.6241 \\
\hline 343.15 & 30 & 983.20 & 1.7654 \\
\hline 343.15 & 40 & 990.11 & 1.9172 \\
\hline 353.15 & 0.1 & 949.93 & 1.1765 \\
\hline 353.14 & 1 & 950.79 & 1.1873 \\
\hline 353.15 & 5 & 954.45 & 1.2326 \\
\hline 353.15 & 10 & 958.87 & 1.2925 \\
\hline 353.15 & 15 & 963.11 & 1.3512 \\
\hline 353.15 & 20 & 967.18 & 1.4115 \\
\hline 353.15 & 30 & 974.89 & 1.5345 \\
\hline 353.15 & 40 & 982.09 & 1.6616 \\
\hline 363.15 & 0.1 & 940.41 & 1.0320 \\
\hline 363.15 & 1 & 941.30 & 1.0410 \\
\hline 363.15 & 5 & 945.18 & 1.0811 \\
\hline 363.15 & 10 & 949.83 & 1.1328 \\
\hline 363.15 & 15 & 954.28 & 1.1834 \\
\hline 363.15 & 20 & 958.54 & 1.2363 \\
\hline 363.15 & 30 & 966.60 & 1.3434 \\
\hline 363.15 & 40 & 974.10 & 1.4533 \\
\hline
\end{tabular}

The combined expanded uncertainties $U_{\mathrm{c}}(k=2)$ were $U_{\mathrm{c}}(T)=12$ $\mathrm{mK}, U_{\mathrm{c}}(p)=0.12 \mathrm{MPa}$ and $U_{\mathrm{c}}(\eta)=2 \%$ with confidence level of 0.95 ${ }^{\mathrm{a}} \rho_{\text {cal }}$ was the density value calculated from Comuñas et al. [10] 